\title{
Reported populations of Meloidogyne ethiopica in Europe identified as Meloidogyne luci
}

Barbara Gerič Stare, ${ }^{\dagger}$ Polona Strajnar, Nik Susič, Gregor Urek, and Saša Širca, Agricultural Institute of Slovenia, Plant Protection Department, 1001 Ljubljana, Slovenia

\begin{abstract}
The tropical group of root-knot nematodes $(\mathrm{RKN})$ including Meloidogyne ethiopica and $M$. luci is a highly polyphagus and damaging group of agricultural pests. M. ethiopica has been detected in several European countries (Slovenia, Italy, Greece) and also in Turkey. However, a description of a new sister species $M$. luci calls for reclassification of all European and Turkish M. ethiopica populations reported up to date as M. luci. Accurate identification can be accomplished through analysis of the esterase isozyme pattern, which is the most distinguishing character between the two otherwise very similar species. Both species display a three banded esterase pattern where the upper band is slightly shifted between the two

species. In addition, molecular characterization of M. ethiopica and M. luci populations revealed that the ITS, SSU, and LSU of the rDNA regions are not appropriate markers for studying relationships among the tropical group of RKNs. However, the COII/IRNA region on mtDNA proved to be very useful for analyzing the phylogenetic relationship of these very closely related species/populations. Mitochondrial sequences with low levels of heteroplasmy allowed clustering of all $\mathrm{M}$. luci populations in a monophyletic clade with a clear separation of this recently described species from $M$. ethiopica. At the same time, a very close relationship between M. ethiopica and M. luci was confirmed again.
\end{abstract}

Root knot nematodes (RKNs) of the genus Meloidogyne represent one of the most damaging groups of agricultural pests and have been involved in substantial economic loss. The genus consists of approximately 100 nominal species that are obligatory parasites of almost every vascular plant including important agricultural crops. The most damage is caused by the group of four "major" species, $M$. incognita, $M$. arenaria, M. javanica, and M. hapla, which are the most distributed worldwide. Additionally, several other RKNs may become emerging pests in the future of agriculture because of intensive global trade, changes in production technologies involving reduced pesticide usage, and climate changes. The emerging species mostly belong to the tropical RKNs group, which reproduce parthenogenetically. They exhibit a wide host range and great damage potential but have limited distribution. One such pest is M. ethiopica, which was listed among emerging global agricultural pests (Hunt and Handoo 2009) and added to the EPPO Alert list of harmful organisms in 2011. M. ethiopica was described by Whitehead in 1968 from the Mlalo region of Tanzania. It is currently distributed in Africa, South America, Asia, and southern Europe. It has been reported to have been found in Kenya, Ethiopia, Mozambique, Zimbabwe, Tanzania, South Africa (Whitehead 1968, 1969), Chile, Brazil, Peru (Carneiro et al. 2003, 2004), Slovenia (Širca et al. 2004), Greece (Conceição et al. 2012), Turkey (Aydınlı et al. 2013), and Italy (Maleita et al. 2012). M. ethiopica can parasitize several crops including monocotyledons, dicotyledons, and herbaceous and woody plants (Carneiro et al. 2003, 2004; Lima et al. 2009; O'Bannon 1975; Strajnar et al. 2009; Whitehead 1968). It is widely distributed in vineyards in Chile (Aballay et al. 2009). M. ethiopica has been reported to reduce yield quality and quantity in kiwi (Actinidia deliciosa) in Brazil (Carneiro et al. 2003) and in grapevine (Vitis vinifera) in Chile (Carneiro et al. 2007). Medina et al. (2014) reported M. ethiopica on potato plants from Parana State, Brazil, showing root systems with multiple galls and tubers with protuberances.

\section{${ }^{\dagger}$ Corresponding author. E-mail: barbara.geric@kis.si}

*The $\boldsymbol{e}$-Xtra logo stands for "electronic extra" and indicates that two supplementary tables and three supplementary figures are published online.

Accepted for publication 21 May 2017.

(c) 2017 The American Phytopathological Society
Correct species identification is needed for appropriate pest management. RKN species identification is based on a combination of morphometrical, biochemical, and molecular methods. $M$. ethiopica species identification using classical morphology requires a detailed analysis of morphological features of the female, male, and secondstage juveniles. However, the species is very difficult to distinguish from the sibling species $M$. inornata and the recently described M. luci (Carneiro et al. 2014) because of morphometrical similarity and intraspecific variation in the group. Besides, the perineal patterns of $M$. ethiopica are highly variable and similar to M. luci (Carneiro et al. 2014) and were characterized as varying from the $M$. arenaria type to the "acrita" type of M. incognita (Golden 1992). M. luci has been reported to have been found in Brazil, Chile, Iran (Carneiro et al. 2014), Guatemala (Janssen et al. 2016), and Portugal (Maleita et al. 2016). Analyzing phenotypes of malate dehydrogenase (MDH) and esterase (EST) isozymes has proved to be a useful approach in RKN species identification (Esbenshade and Triantaphyllou 1985, 1990). Besides morphometrical and biochemical analyses, molecular tools are also used for nematode species determination. Several molecular markers have been used for phylogenetic studies of nematodes from the order Tylenchida and genus Meloidogyne. Holterman et al. (2009) reported that the small subunit ribosomal DNA (SSU rDNA) was a good phylogenetic marker for the phylum Nematoda as well as a good species identification marker for Tylenchidae. However, that does not hold for most of the tropical group RKN species that include M. ethiopica (Holterman et al. 2006, 2009; Kiewnick et al. 2014) because of high intraspecific variation.

In this study, we have re-examined M. ethiopica populations from Slovenia, Greece, Turkey, Brazil, and South Africa and compared them with a population of $M$. luci from Iran to assess whether the closely related $M$. luci and M. ethiopica populations can be unambiguously distinguished by differential host, isozyme pattern, and/or molecular marker regions of rDNA and mtDNA widely used in the phylum Nematoda. The present study provides information on the biological, biochemical, and molecular features of $M$. luci and M. ethiopica populations from the different continents: South America, Africa, Europe, and Asia.

\section{Materials and Methods}

Nematode populations. Nematode populations were collected from different countries and continents where M. ethiopica and M. luci occur (Supplementary Table S1). Populations from Slovenia, Greece, and Turkey previously identified as M. ethiopica (Aydınlı et al. 
2013; Conceição et al. 2012; Strajnar et al. 2009; Širca et al. 2004) are reclassified in this study to $M$. luci. All populations were maintained on tomato cultures cv. Cuore di Bue planted in sterile sand and kept in a greenhouse at 20 to $25^{\circ} \mathrm{C}$. Nematodes used for analyses were isolated from the fresh cultures. Using a dissecting microscope, females and egg masses were isolated from infested roots with a scalpel and a nematological needle. Males were isolated from the sand by the decanting method (Hržič 1973) followed by Baerman's funnel extraction.

Differential host status. Differential host test was based on the Taylor and Sasser (1978) method. The experiment was set up in the greenhouse at an average temperature of $22^{\circ} \mathrm{C}$. Two populations of M. ethiopica from Brazil and Africa, and one population of $M$. luci from Slovenia, were chosen in order to represent the esterase phenotypes, E3, E2, and L3, respectively. They were tested for reproduction on watermelon cv. Charleston Gray. Seven-week-old plants were inoculated with an aqueous suspension containing 3,000 RKN eggs obtained from the cultures as described by Hussey and Barker (1973). The experiment was terminated 60 days after inoculation and the final nematode population was determined. Five test plants of each tested RKN population were evaluated for the nematode reproduction rate.

Host suitability was assessed by determining the reproduction factor $\mathrm{R}=\mathrm{Pf} / \mathrm{Pi}$ (final population of nematode eggs and juveniles divided by the number of inoculated nematode eggs). $\mathrm{R}$ value defined four plant categories: $\mathrm{R}>10=$ excellent host; $10>\mathrm{R}>1$ = good host; $\mathrm{R}$ close to 1 = maintenance host, and $\mathrm{R}<1=$ poor host to non-host (Ferris et al. 1993).

Isoenzymes. Isolated egg-laying females of $M$. luci and M. ethiopica populations were rinsed with reagent-grade water and transferred to an 8-sample-well stamp placed in an ice bath. A single female was placed in a separate sample well containing $1.3 \mu \mathrm{l}$ of extraction buffer (20\% sucrose, $2 \%$ Triton X-100, $0.01 \%$ bromophenol blue) (Esbenshade and Triantaphyllou 1985) and macerated with a needle to release the body contents. Samples were loaded on $8 / 0.5$ sample applicators, which were placed into the applicator arms of the PhastSystem equipment (GE Healthcare Life Sciences; Pharmacia). Freshly isolated $M$. javanica females (a culture obtained from the Plant Protection Service, Wageningen, The Netherlands) were used as a reference. PhastGel gradient gel ( 8 to 25 ) with a buffer system was used according to manufacturer's instructions. The conditions of electrophoresis and gel staining for MDH (EC 1.1.1.37) and EST (EC 3.1.1.1) activity were as described by Karssen et al. (1995). Enzymatic reaction was stopped by rinsing the gel with distilled water and fixed for $5 \mathrm{~min}$ in a solution of $10 \%$ acetic acid, $10 \%$ glycerol, and $80 \%$ distilled water. Relative migration rates (Rm) of EST bands were calculated as a ratio of migration distance of EST bands to the upper $M$. javanica EST band.

DNA extraction. DNA was extracted from a single egg mass of each population. A Promega Genomic DNA Wizard purification kit (Madison, WI) was used according to manufacturer's instructions. Extracted DNA was diluted in $10 \mu \mathrm{l}$ of DNA rehydration solution (10 mM Tris-HCl, $1 \mathrm{mM}$ EDTA).

PCR amplification. SSU rDNA fragments were amplified with two set of primers, 1A (5'-GGCGATCGAAAAGATTAAGCC-3') and 3B (5'-GGCGATCGATTGGCAAATGCTTTCGC-3') described by Baldwin et al. (1997), and MelF (5'-TACGGACTGAGA TAATGGT-3') and MelR (5'-GGTTCAAGCCACTGCGA-3') described by Tigano et al. (2005). Both primers sets gave overlapping sequences and resulted in an SSU rDNA gene sequence of approximately $1,700 \mathrm{bp}$. PCR reactions contained $1 \mu \mathrm{l}$ of isolated DNA, $10 \mathrm{mM}$ Tris- $\mathrm{HCl} \mathrm{pH} 8.3,25 \mathrm{mM} \mathrm{MgCl} 2,2.5 \mathrm{mM}$ of each of the dNTPs, $1 \mu \mathrm{M}$ of each primer, 1U GoTaq DNA Polymerase (Promega), and distilled water up to $25 \mu \mathrm{l}$. The amplification was carried out in a thermal cycler Veriti (Applied Biosystems) using the following program: initial denaturation at $94^{\circ} \mathrm{C}$ for $3 \mathrm{~min} ; 40$ cycles of denaturation at $94^{\circ} \mathrm{C}$ for $30 \mathrm{~s}$, annealing at $57^{\circ} \mathrm{C}$ for $45 \mathrm{~s}$, and elongation at $72^{\circ} \mathrm{C}$ for $2 \mathrm{~min}$; followed by a final extension at $72^{\circ} \mathrm{C}$ for $7 \mathrm{~min}$.

The region of mtDNA, located in the $3^{\prime}$ portion of the gene that codes for cytochrome oxidase subunit II (COII) through a portion of the 16S rRNA (IRNA) gene, was amplified with primers $\mathrm{C} 2 \mathrm{~F} 3$ 5'-GGTCAATGTTCAGAAATTTGTGG-3' and 1108 5'-TACCT TTGACCAATCACGCT-3' (Powers and Harris 1993). PCR reactions contained $1 \mu \mathrm{l}$ of isolated DNA (see above), $10 \mathrm{mM}$ Tris$\mathrm{HCl} \mathrm{pH} \mathrm{8.3,} 33 \mathrm{mM} \mathrm{MgCl}_{2}, 2.5 \mathrm{mM}$ of each of the dNTPs, $1 \mu \mathrm{M}$ of each of the primers, 1U GoTaq DNA Polymerase (Promega), and distilled water up to $25 \mu \mathrm{l}$. The amplification was carried out in a thermal cycler Veriti (Applied Biosystems) using the following program: initial denaturation at $93^{\circ} \mathrm{C}$ for $3 \mathrm{~min} ; 33$ cycles of denaturation at $93^{\circ} \mathrm{C}$ for $4 \mathrm{~s}$, combined annealing and elongation at $48^{\circ} \mathrm{C}$ for $8 \mathrm{~min}$; followed by a final extension at $48^{\circ} \mathrm{C}$ for $10 \mathrm{~min}$.

A D2-D3 fragment of LSU rRNA gene was amplified using D2A (5'-ACAAGTACCGTGAGGGAAAGTTG-3') and D3B (5'-TCGG AAGGAACCAGCTACTA-3') primers (Rubtsova et al. 2001) while the ITS1-5.8S-ITS2 region of rRNA was amplified using forward $5^{\prime}$ TTGATTACGTCCCTGCCCTTT-3' (Vrain et al. 1992) and reverse 5'-TCCTCCGCTAAATGATATG-3' (Ferris et al. 1993) primers. The PCR conditions were the same as for the amplification of SSU rDNA fragments. Electrophoresis of all amplified DNA products was performed on a $1 \%$ TBE agarose gel.

Transformation, isolation of plasmid DNA, and sequencing. SSU rDNA and mtDNA PCR products, and PCR products of selected populations for ITS and LSU rDNA regions, were transformed using a pGEM-T Easy Vector System II (Promega) according to the manufacturer's instructions. Plasmid DNA was isolated by JetQuick Plasmid Miniprep (Genomed) following the manufacturer's instructions. Macrogen Inc. (Korea) provided sequencing of plasmids with universal primers SP6 and T7, while the amplicons of LSU rDNA and ITS regions (except for the populations that were cloned) were sequenced directly with the primers used in amplification.

Sequence analysis. DNA sequences were assembled using the BioEdit v. 7.0.5.2 software (T. A. Hall). An alignment was prepared with EMBL-EBI Multiple Sequence Alignment Tools MUSCLE (http://www.ebi.ac.uk/Tools/msa/muscle/) and edited manually if necessary. To assess relationships between populations and related species, DNA sequences determined in this study and sequences from the NCBI GenBank public database were included in the analyses. The neighbor joining (NJ) method conducted in MEGA v. 6.06 (K. Tamura et al.), maximum likelihood (ML) method in PhyML 3.0 (S. Guindon et al.; http://www.atgc-montpellier.fr/), and Bayesian inference method in MrBayes v. 3.2.2. (F. Ronquist and J. P. Huelsenbeck) were all used for phylogenetic and molecular evolution analyses. Bayesian phylogeny inferences in MrBayes and the ML analysis in PhyML implemented a substitution model selected according to the Akaike information criterion calculated with the software jModelTest v. 2.1.4. (D. Daribba et al.). Model parameters used for the NJ method were determined with MEGA 6. An analysis of five million generations based on four Markov chains sampled every 100 generations was performed with MrBayes. Calculation of the $50 \%$ majority rule consensus tree ignored the first $0.5 \%$ of trees. A burn-in of $25 \%$ was used when the $50 \%$ majority rule consensus tree was calculated to receive posterior probabilities.

\section{Results}

Differential host status. Watermelon cv. Charleston Gray was a poor host for populations of M. ethiopica and M. luci. All three RKN populations of M. ethiopica and $M$. luci had a reproduction factor under 1. The Brazilian and African populations of M. ethiopica and the Slovenian population of $M$. luci had Rf of $0.3,0.7$, and 0.6 , respectively.

Isoenzymes. MDH phenotype $\mathrm{N} 1$ was determined in all the M. ethiopica and M. luci populations that were examined. The EST phenotype E3 was determined in the Brazilian M. ethiopica population, while the phenotype E2 was exhibited in the South African M. ethiopica population. The two banded phenotype E2 differs from E3 by the absence of the middle band of E3. All M. luci populations, including populations from Slovenia, Greece, and Turkey, previously identified and reported as M. ethiopica with E3 EST pattern, displayed EST phenotype L3. Phenotypes E3 and L3 differed in the Rm of the upper EST band of 0.96 and 1.00, respectively (Fig. 1, Table 1). 
Sequence variability and comparison. All sequences obtained in this study were deposited at the European Nucleotide Archive, EMBL Nucleotide Sequence Database with the accession numbers LN626920-LN626967 and LN713293-LN713302. Phylogenetic relationships were evaluated between $M$. ethiopica and M. luci populations from different regions and compared with related Meloidogyne species using four molecular markers: SSU rDNA, LSU rDNA, ITS rDNA, and COII/IRNA mtDNA.

Low variation with $99.6 \%$ sequence identity was observed in the mtDNA region COII/IRNA among $M$. luci sequences from this study and other sequences available in the database. A slightly higher level of variation (99.3\% sequence identity) was observed in a combined set of M. ethiopica and M. luci sequences (Supplementary Table S2). Considerably higher variation was observed in the three rDNA markers. Sequence identities for $M$. luci sequences from this study and other available sequences in the database are 97.8, 94.8, and $89.1 \%$ for SSU, LSU, and ITS, respectively (data not shown).

Sequence similarity trees based on mtDNA and rDNA sequences were constructed using NJ, ML, and Bayesian inference methods. Tree topologies were fairly consistent irrespective of the algorithm and are represented by the ML trees (Fig. 2, Supplementary Figs. S1, S2, and S3). Evolutionary relationships of RKN sequences inferred using the mtDNA region of $40 \mathrm{RKN}$ species and populations clustered all M. luci sequences in a monophyletic clade (Fig. 2). On the other hand, trees based on SSU, LSU, and ITS representing 64, 28, and 58 sequences of RKN species and populations do not cluster $M$. luci sequences in monophyletic clades.

Our study comprehensively analyzed rDNA and mtDNA sequences of M. ethiopica and M. luci populations from different countries including sequences of other Meloidogyne spp. from GenBank. The study revealed that the SSU, LSU, and ITS regions of the rDNA are not appropriate markers for studying relationships among M. ethiopica and M. luci populations as well as other species from the RKN tropical group. The sequences of the tropical RKN species M. ethiopica, $M$. luci, $M$. arenaria, $M$. javanica, and $M$. incognita did not form monophyletic groups in any of the rDNA studied markers. Furthermore, genetic variability was greater within the species than between species even on the relatively conserved region SSU.

On the contrary, the COII/IRNA region of mtDNA proved to be very useful for analyzing the phylogenetic relationship of the these very closely related RKN species and populations. Low levels of heteroplasmy in this region allowed the formation of a monophyletic clade for $M$. luci with a clear separation of this recently described

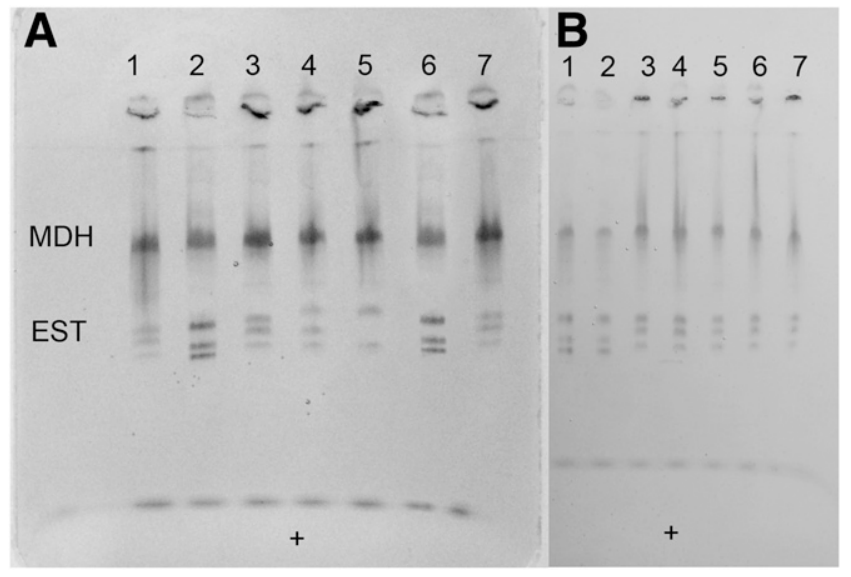

Fig. 1. Isozymes malate dehydrogenase (MDH) and esterase (EST) phenotype patterns of individual female of Meloidogyne luci and M. ethiopica. A, lanes: 1 M. Iuci from Turkey, 3 - M. luci from Slovenia, 4 - M. ethiopica from Brazil, 5 M. ethiopica from South Africa, 7 - M. luci from Greece, M. javanica (lanes 2, 6) as a reference. B, M. javanica (lanes 1, 2), M. luci from Slovenia (lanes 3, 4), and $M$. Iuci from Iran (lanes 5, 6, 7). Populations from Slovenia, Greece, and Turkey have been previously identified as M. ethiopica (Aydınlı et al. 2013; Conceição et al. 2012; Strajnar et al. 2009; Šrca et al. 2004), but are reclassified in this study to M. luci. species. At the same time, a very close relationship between $M$. ethiopica and $M$. luci and also M. inornata was observed.

\section{Discussion}

The characteristics of the newly described species $M$. luci (Carneiro et al. 2014) and our results indicate a very close relationship between the sister species of $M$. luci, M. inornata, and M. ethiopica. Morphologically $M$. luci can be distinguished from other RKNs through several characteristics such as perineal pattern, morphology of the male or female stylet, and male and second stage juvenile head and tail morphology (Carneiro et al. 2014). However, on the basis of morphological features alone, the sister species $M$. luci and M. ethiopica are very difficult to distinguish. For example, the feature of the $M$. luci perineal pattern, which is often used as a diagnostic characteristic, is very similar to $M$. ethiopica (mix of $M$. arenaria and $M$. incognita). Using scanning electron microscopy Carneiro et al. (2014) reported the following morphological differences between $M$. luci and M. ethiopica: the reniform stylet knobs, the cephalic region of males, and the shape of male stylet. For example, in $M$. luci the stylet knobs are small and rounded to a heart shape as compared with the reniform shape of $M$. ethiopica. In fact, all the above mentioned differences are quite difficult to observe using the most widely used light microscopy and even then, highly trained and experienced diagnostic specialists are needed. In addition, most of the morphometrical characteristics of juveniles, females, and males overlap between sister species, thus making morphological identification unreliable.

Host status. Watermelon cv. Charleston Gray was one of the hosts of the North Carolina differential host test, and it was used to determine the host status of the South African and Brazilian populations of M. ethiopica and Slovenian populations of M. luci. All populations in our study had a reproduction factor under 1 and therefore watermelon cv. Charleston Gray was determined to be in the range of a poor to non-host. Our results contradict the finding of Carneiro et al. (2003, 2007) who reported that watermelon cv. Charleston Gray was a good host for M. ethiopica (although the exact Rf was not reported). On the other hand, watermelon cv. Charleston Gray was reported as a non-host for the sister species M. luci (Carneiro et al. 2014). The reported $\mathrm{Rf}=0.3$ in $M$. luci is not different from the $\mathrm{Rf}=0.3$ of the Brazilian M. ethiopica population from this study. Although previously reported data suggests that watermelon $\mathrm{cv}$. Charleston Gray could be used as a differential host between M. ethiopica (Carneiro et al. 2007) and M. luci (Carneiro et al. 2014), our results do not support this.

There is no dependable differential host between M. ethiopica and $M$. luci and it seems that these two species are so closely related that there is no biological difference between the two species in terms of parasitizing different plant species. Based on crop damage and economic losses that $M$. ethiopica has been causing on grapevines and kiwi (Carneiro et al. 2003), M. ethiopica has been included on the EPPO Alert list of harmful organisms in 2011. M. ethiopica is also reported to affect potato production (Medina et al. 2014) and $M$. luci has been shown as a potential threat to the potato production as well (Maleita et al. 2016). Further, $M$. luci has been reported on several vegetables, flowers, and fruits species (Carneiro et al. 2014). It should also be noted that some hosts previously reported for

Table 1. The relative migration rates $(\mathrm{Rm})$ of esterase isozymes of studied Meloidogyne ethiopica and $M$. luci populations and of reference $M$. javanica population

\begin{tabular}{ll}
\hline Species and populations & \multicolumn{1}{c}{$\mathbf{R m}$} \\
\hline M. javanica & $1.00,1.11,1.16$ \\
M. ethiopica Brazil & $0.96,1.05,1.13$ \\
M. ethiopica South Africa & $0.96,1.13$ \\
M. luci Slovenia & $1.00,1.05,1.13$ \\
M. luci Greece & $1.00,1.05,1.13$ \\
M. luci Turkey & $1.00,1.05,1.13$ \\
M. luci Iran & $1.00,1.05,1.13$ \\
\hline
\end{tabular}




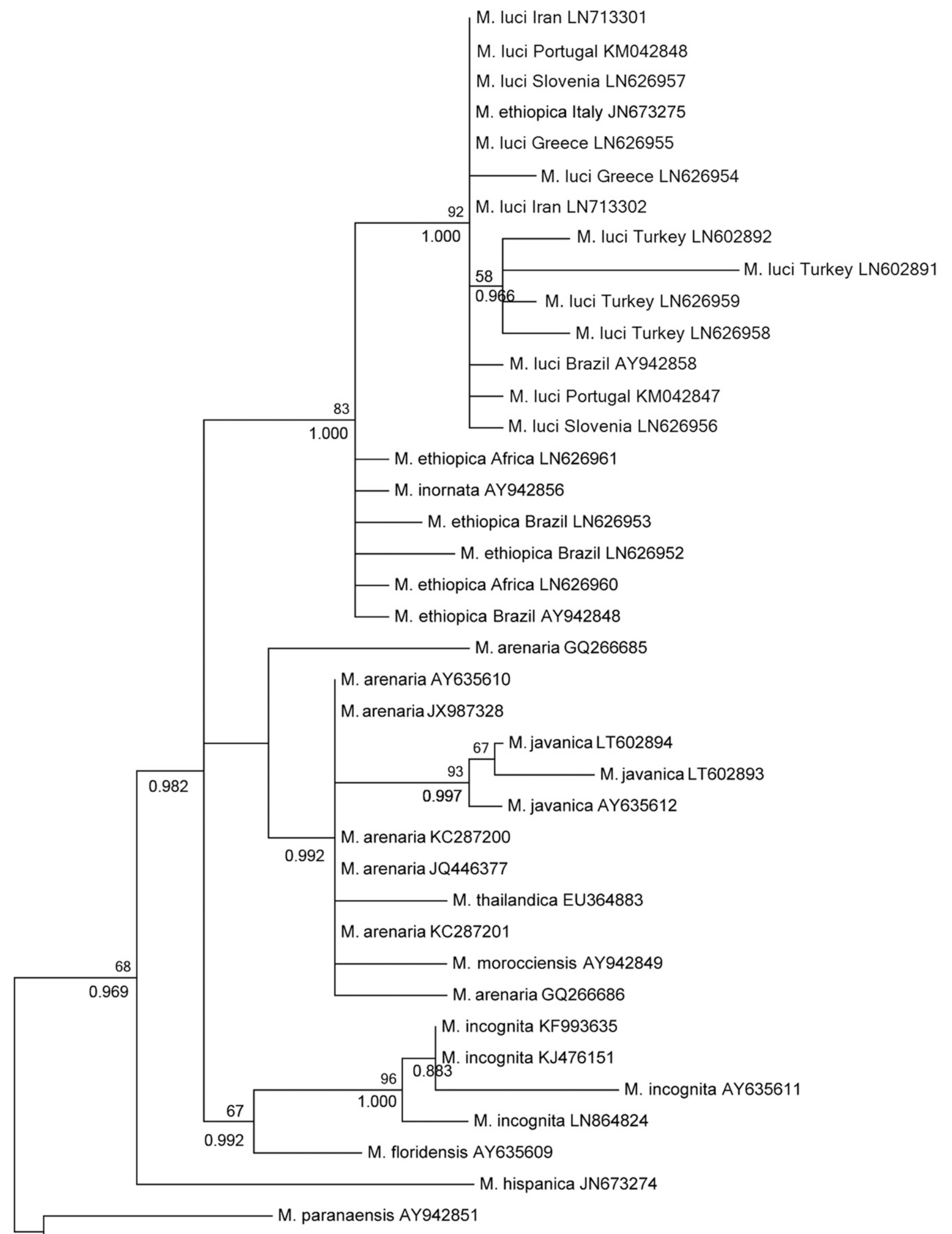

M. arabicida AY942852

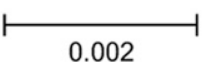

Fig. 2. Evolutionary relationships of Meloidogyne sequences inferred using the mtDNA region between COIl and 16S rRNA genes of 40 Meloidogyne species and populations and the ML method. Bootstrap support over 50\% shown above branches, posterior probabilities from the Bayesian inference above 0.90 shown below branches. 
M. ethiopica (Strajnar et al. 2009) are in fact hosts of M. luci, as the identity of Slovene $M$. ethiopica population has been reclassified into M. luci. In addition, the potential of $M$. ethiopica to survive outdoors under a continental climate even in areas where soil temperatures fall below zero during winter, as well as under a sub-Mediterranean climate (Strajnar et al. 2011) now applies to $M$. luci. This indicates that $M$. luci could establish and spread in the southern and central parts of the EPPO region. Based on the potential threat $M$. luci represents for agriculture in the EPPO region, it is the opinion of the authors that $M$. luci should be added to the national and international lists of harmful organisms of phytosanitary concern.

Isozyme profile. Carneiro et al. $(2004,2007)$ reported that isozyme phenotyping is currently the most useful procedure for differentiation of $M$. ethiopica from other species as the esterase phenotype E3 is species-specific for M. ethiopica. However, E3 is not the only esterase phenotype found in M. ethiopica. Mandefro and Dagne (2000) previously reported two esterase phenotypes, E2 and E3, for M. ethiopica but the relative migration rates were not reported. While some Meloidogyne species have one species-specific esterase pattern, some species like M. arenaria (Esbenshade and Triantaphyllou 1985), M. incognita (Carneiro et al. 2000; Esbenshade and Triantaphyllou 1985), and M. exigua (Muniz et al. 2008) are known to display several esterase patterns. Some patterns can be found in more than one species; for example, M. morocciensis (Rammah and Hirschmann 1990) had the same esterase phenotype as $M$. arenaria (A3). In this study we have shown that Slovene, Greek, and Turkish RKN populations previously identified as M. ethiopica and reported to have esterase phenotype E3 (Aydınlı et al. 2013; Conceição et al. 2012; Strajnar et al. 2009) in fact exhibit a three banded esterase pattern where the upper band is slightly lower than in M. ethiopica populations from Brazil, Chile, and Peru. This pattern has recently been recognized as L3 and specific for a newly described species $M$. luci (Carneiro et al. 2014). A close look at the published esterase profile of the $M$. ethiopica from Italy reveals that this population also has the L3 esterase phenotype, although it was reported as an E3 esterase phenotype (Maleita et al. 2012). Therefore, all previously reported populations of M. ethiopica in Europe and Turkey in fact belong to the recently described $M$. luci. This misidentification is the consequence of the fact that L3 and E3 phenotypes differ in migration rate of one band only, which is hard to spot unless populations with both phenotypes are analyzed on the same gel.

Isozyme MDH pattern does not differentiate between $M$. ethiopica and $M$. luci as phenotype N1 was determined in all tested populations, which is in accordance with previous reports (Carneiro et al. 2007, 2014; Strajnar et al. 2009).

Sequences, phylogeny, and evolution. The majority of tropical RKNs, including $M$. ethiopica and $M$. luci, reproduce by obligate mitotic parthenogenesis (Carneiro et al. 2004, 2014; CastagnoneSereno and Danchin 2014). High diversity, even within individual nematodes, has previously been reported for the ITS region in $M$. arenaria, M. javanica, and M. incognita by Hugall et al. (1999). Based on the high variability of ITS sequences in tropical Meloidogyne that reproduce with obligate mitotic parthenogenesis, Hugall et al. (1999) hypothesized that these species have hybrid origin and reticulate evolution. Recent comparative genomics studies of three RKN species confirmed hybrid origin of apomictic RKNs (Lunt et al. 2014). Taking into account the hybrid origin of apomictic RKNs, finding high variability of rDNA regions in $M$. luci is not unexpected. However, high genetic variability represents a challenge for the description of a new species in the group of tropical RKNs as well as for the development of identification tools. While mtDNA in apomictic RKNs can be used for species identification (Janssen et al. 2016; Pagan et al. 2015), it cannot be used to study species evolution within the group as the reticulate evolution of these species was very complex (Lunt et al. 2014). While we observed a close relationship between M. ethiopica, M. luci, and $M$. inornata based on COII/IRNA region of mtDNA, a close relationship between these three species has also been shown on coding mtDNA regions by Janssen et al. (2016).

Since variability of both the rDNA as well as the mtDNA regions has been well documented in this and previous studies (Holterman et al. 2006, 2009, 2012; Kiewnick et al. 2014; Powers et al. 2005;
Tigano et al. 2005), careful selection of molecular markers to study phylogenetic relationships or to confirm the identification of Meloidogyne species is needed. Our study suggests that mtDNA is suitable for $M$. luci species identification, while the SSU rRNA gene, ITS region, and a segment of LSU rDNA were revealed as poor identification markers for tropical Meloidogyne species identification. Analyzing phenotypes of MDH and EST isozymes in combination with phylogenetic analysis of mtDNA should be used to distinguish between $M$. ethiopica and $M$. luci. In the study by Janssen et al. (2016), M. luci from Slovenia and Iran shared the same haplotype, while $M$. luci from Guatemala exhibited another haplotype occurring in a paraphyletic position. It seems that genomic approach will be needed to resolve phylogenetic relationships of closely related populations of $M$. ethiopica, M. luci, and M. inornata.

\section{Acknowledgments}

This work was financially supported by the Slovenian Research Agency in the frame of Research Programme Agrobiodiversity (P4-0072). Part of this work was realized within the frame of FP7 Project CropSustaIn, grant agreement FP7REGPOT-CT2012-316205. The authors are grateful to Dr. Gerrit Karssen, Dr. Isabel Luci Pisa Mata da Conceição, Dr. Gökhan Aydınlı, and Dr. Mariette Marais for providing Meloidogyne populations used in this study, to Dr. Hans-Josef Schroers for the discussions on phylogenetic analysis, and to Dr. Vivian Blok for discussion on RKNs.

\section{Literature Cited}

Aballay, E., Persson, P., and Mårtensson, A. 2009. Plant-parasitic nematodes in Chilean vineyards. Nematropica 39:85-97.

Aydınlı, G., Mennan, S., Devran, Z., Šrca, S., and Urek, G. 2013. First report of the root-knot nematode Meloidogyne ethiopica on tomato and cucumber in Turkey. Plant Dis. 97:1262.

Baldwin, J. G., Frisse, L. M., Vida, J. T., Eddleman, C. D., and Thomas, W. K. 1997. An evolutionary framework for the study of developmental evolution in a set of nematodes related to Caenorhabditis elegans. Mol. Phylogenet. Evol. 8:249-259.

Carneiro, R. M. D. G., Almeida, M. R. A., Cofcewicz, E. T., Magunacelaya, J. C., and Aballay, E. 2007. Meloidogyne ethiopica, a major root-knot nematode parasitising Vitis vinifera and other crops in Chile. Nematology 9:633-639.

Carneiro, R. M. D. G., Almeida, M. R. A., and Queneherve, P. 2000. Enzyme phenotypes of Meloidogyne spp. populations. Nematology 2:645-654.

Carneiro, R. M. D. G., Correa, V. R., Almeida, M. R. A., Gomes, A. C. M. M. Deimi, A. M., Castagnone-Sereno, P., and Karssen, G. 2014. Meloidogyne luci n. sp. (Nematoda: Meloidogynidae), a root-knot nematode parasitising different crops in Brazil, Chile and Iran. Nematology 16:289-301.

Carneiro, R. M. D. G., Gomes, C. B., Almeida, M. R. A., Gomes, A. C. M. M., and Martins, I. 2003. First record of Meloidogyne ethiopica Whitehead, 1968 on kiwi in Brazil and reaction on different plant species. Nematol. Bras. 27:151-158.

Carneiro, R. M. D. G., Randing, O., Almeida, M. R. A., and Gomes, A. C. M. M 2004. Additional information on Meloidogyne ethiopica Whitehead, 1968 (Thylenchida: Meloidogynidae) a root-knot nematode parasitising kiwi fruit and grape-vine from Brazil and Chile. Nematology 6:109-123.

Castagnone-Sereno, P., and Danchin, E. G. J. 2014. Parasitic success without sex the nematode experience. J. Evol. Biol. 27:1323-1333.

Conceição, I. L., Tzortzakakis, E. A., Gomes, P., Abrantes, I., and da Cunha, M. J. 2012. Detection of the root-knot nematode Meloidogyne ethiopica in Greece. Eur. J. Plant Pathol. 134:451-457.

Esbenshade, P. R., and Triantaphyllou, A. C. 1985. Use of isozyme phenotypes for identification of Meloidogyne species. J. Nematol. 17:6-20.

Esbenshade, P. R., and Triantaphyllou, A. C. 1990. Isozyme phenotypes for the identification of Meloidogyne species. J. Nematol. 22:10-15.

Ferris, V. R., Ferris, J. M., and Faghihi, J. 1993. Variation in spacer ribosomal DNA in some cyst-forming species of plant parasitic nematodes. Fundam. Appl. Nematol. 16:177-184

Golden, A. M. 1992. Large phasmids in the female of Meloidogyne ethiopica Whitehead. Fundam. Appl. Nematol. 15:189-191.

Holterman, M., Karssen, G., van den Elsen, S., van Megen, H., Bakker, J., and Helder, J. 2009. Small subunit rDNA-based phylogeny of the Tylenchida sheds light on relationships among some high-impact plant-parasitic nematodes and the evolution of plant feeding. Nematology 99:227-235.

Holterman, M., van der Wurff, A., van den Elsen, S., van Megen, H., Bongers, T., Holovachov, O., Bakker, J., and Helder, J. 2006. Phylum-wide analysis of SSU rDNA reveals deep phylogenetic relationships among nematodes and accelerated evolution toward crown clades. Mol. Biol. Evol. 23:1792-1800.

Holterman, M. H. M., Oggenfuss, M., Ernst Frey, J., and Kiewnick, S. 2012 Evaluation of high-resolution melting curve analysis as a new tool for rootknot nematode diagnostics. J. Phytopathol. 160:59-66.

Hržič, A. 1973. Extraction of nematodes from soil with whirling motion. Zašt Bilja. 122:53-60. 
Hugall, A., Stanton, J., and Moritz, C. 1999. Reticulate evolution and the origins of ribosomal internal transcribed spacer diversity in apomictic Meloidogyne. Mol. Biol. Evol. 16:157-164.

Hunt, D. J., and Handoo, Z. A. 2009. Taxonomy, identification and principal species. Pages 55-88 in: Root-knot Nematodes. R. N. Perry, M. Moens, and J. L. Starr, eds. CABI, Wallingford, U.K.

Hussey, R. S., and Barker, K. R. 1973. A comparison of methods of collecting inocula of Meloidogyne spp. including a new technique. Plant Dis. Rep. 57: 1025-1028.

Janssen, T., Karssen, G., Verhaeven, M., Coyne, D., and Bert, W. 2016. Mitochondrial coding genome analysis of tropical root-knot nematodes (Meloidogyne) supports haplotype based diagnostics and reveals evidence of recent reticulate evolution. Sci. Rep. 6:22591.

Karssen, G., Van Hoenselaar, T., Verkerk-Bakker, B., and Janssen, R. 1995. Species identification of cyst and root-knot nematodes from potato by electrophoresis of individual females. Electrophoresis 16:105-109.

Kiewnick, S., Holterman, M., van den Elsen, S., van Megen, H., Frey Juerg, E., and Helder, J. 2014. Comparison of two short DNA barcoding loci (COI and COII) and two longer ribosomal DNA genes (SSU \& LSU rRNA) for specimen identification among quarantine root-knot nematodes (Meloidogyne spp.) and their close relatives. Eur. J. Plant Pathol. 140:97-110.

Lima, E. A., Mattos, J. K., Moita, A. W., Carneiro, R. G., and Carneiro, R. M. D. G. 2009. Host status of different crops for Meloidogyne ethiopica control. Trop. Plant Pathol. 34:152-157.

Lunt, D. H., Kumar, S., Koutsovoulos, G., and Blaxter, M. L. 2014. The complex hybrid origins of the root knot nematodes revealed through comparative genomics. PeerJ 2:e356.

Maleita, C. M., Esteves, I., Cardoso, J. M. S., José Cunha, M., Carneiro, R. M. D. G., and Abrantes, I. 2016. First report of Meloidogyne luci in Portugal - a potential threat to the potato crop. Page 153 in: 32nd European Society of Nematologists Symposium Abstract Book, Braga, Portugal. Published online (accessed 5 October 2016). https://www.esn-online.org/core/js/tinymce/plugins/moxiemanager/data/files/ ESN2016_Abstract_Book.pdf

Maleita, C. M., José Simões, M., Egas, C., Curtis, R. H. C., and Abrantes, I. M. O. 2012. Biometrical, biochemical, and molecular diagnosis of Portuguese Meloidogyne hispanica isolates. Plant Dis. 96:865-74.

Mandefro, W., and Dagne, K. 2000. Cytogenetic and esterase isozyme variation of root-knot nematode populations from Ethiopia. Afr. J. Plant Prot. 10:39-47.

Medina, I. L., Coila, V. H. C., Gomes, C. B., Pereira, A. S., and Nazareno, N. R. X. 2014. Meloidogyne ethiopica report in Parana state, Brazil, and reaction of potato cultivars to root-knot nematode. Hortic. Bras. 32:482-485.
Muniz, M. F. S., Campos, V. P., Castagnone-Sereno, P., Castro, J. M. C., Almeida, M. R. A., and Carneiro, R. M. D. G. 2008. Diversity of Meloidogyne exigua (Tylenchida: Meloidogynidae) populations from coffee and rubber tree. Nematology 10:897-910.

O'Bannon, J. H. 1975. Nematode survey in Ethiopia. Institute of Agricultural Research. FAO, Rome, Addis Ababa, Ethiopia.

Pagan, C., Coyne, D., Carneiro, R., Kariuki, G., Luambano, N., Affokpon, A., and Williamson, V. M. 2015. Mitochondrial haplotype-based identification of ethanol-preserved root-knot nematodes from Africa. Phytopathology 105:350-357.

Powers, T. O., and Harris, T. S. 1993. A polymerase chain reaction method for identification of five major Meloidogyne species. J. Nematol. 25:1-6.

Powers, T. O., Mullin, P. G., Harris, T. S., Sutton, L. A., and Higgins, R. S. 2005 Incorporating molecular identification of Meloidogyne spp. into a large-scale regional nematode survey. J. Nematol. 37:226-235.

Rammah, A., and Hirschmann, H. 1990. Meloidogyne morocciensis n. sp. (Meloidogyninae), a root-knot nematode from Morocco. J. Nematol. 22:279-291.

Rubtsova, T. V., Subbotin, S. A., Brown, D. J. F., and Moens, M. 2001. Description of Longidorus sturhani sp. nov. (Nematoda: Longidoridae) and molecular characterization of several Longidorid species from Western Europe. Russ. J. Nematol. 9:127-136.

Širca, S., Urek, G., and Karssen, G. 2004. First report of the root-knot nematode Meloidogyne ethiopica on tomato in Slovenia. Plant Dis. 88:680.

Strajnar, P., Sirca, S., Gerič Stare, B., and Urek, G. 2009. Characterisation of the root-knot nematode, Meloidogyne ethiopica Whitehead, 1968, from Slovenia Russ. J. Nematol. 17:135-142.

Strajnar, P., Širca, S., Knapič, M., and Urek, G. 2011. Effect of Slovenian climatic conditions on the development and survival of the root-knot nematode Meloidogyne ethiopica. Eur. J. Plant Pathol. 129:81-88.

Taylor, A. L., and Sasser, I. N. 1978. Biology, identification and control of rootknot nematodes (Meloidogyne species). North Carolina State University/ USAID. North Carolina State University Graphics, Raleigh, NC.

Tigano, M., Carneiro, R. M. D. G., Dickson, D. W., and Adams, B. 2005 Phylogeny of Meloidogyne spp. based on SSU rDNA and the intergenic region of mitochondrial DNA sequences. Nematology 7:851-862.

Vrain, T. C., Wakarchuk, D. A., Levesque, A. C., and Hamilton, R. I. 1992. Intraspecific rDNA restriction fragment length polymorphism in the Xiphinema americanum group. Fundam. Appl. Nematol. 15:563-573.

Whitehead, A. G. 1968. Taxonomy of Meloidogyne (Nematoda: Heteroderidae) with description of four new species. Trans. Zool. Soc. Lond. 31:263-401.

Whitehead, A. G. 1969. The distribution of root-knot nematodes (Meloidogyne spp.) in tropical Africa. Nematologica 15:315-333. 